ESAIM: M2AN

Vol. 41, No 3,2007 , pp. 447-459

DOI: $10.1051 / \mathrm{m} 2 \mathrm{an}: 2007030$
ESAIM: Mathematical Modelling and Numerical Analysis

www.edpsciences.org/m2an

\title{
MATHEMATICAL FRAMEWORK FOR CURRENT DENSITY IMAGING DUE TO DISCHARGE OF ELECTRO-MUSCULAR DISRUPTION DEVICES
}

\author{
Jeehyun LeE ${ }^{1}$, Jin Keun SeO ${ }^{2}$ And Eung Je Woo ${ }^{3}$
}

\begin{abstract}
Electro-muscular disruption (EMD) devices such as TASER M26 and X26 have been used as a less-than-lethal weapon. Such EMD devices shoot a pair of darts toward an intended target to generate an incapacitating electrical shock. In the use of the EMD device, there have been controversial questions about its safety and effectiveness. To address these questions, we need to investigate the distribution of the current density $\mathbf{J}$ inside the target produced by the EMD device. One approach is to develop a computational model providing a quantitative and reliable analysis about the distribution of $\mathbf{J}$. In this paper, we set up a mathematical model of a typical EMD shock, bearing in mind that we are aiming to compute the current density distribution inside the human body with a pair of inserted darts. The safety issue of TASER is directly related to the magnitude of $|\mathbf{J}|$ at the region of the darts where the current density $\mathbf{J}$ is highly concentrated. Hence, fine computation of $\mathbf{J}$ near the dart is essential. For such numerical simulations, serious computational difficulties are encountered in dealing with the darts having two different very sharp corners, tip of needle and tip of barb. The boundary of a small fishhook-shaped dart inside a large computational domain and the presence of corner singularities require a very fine mesh leading to a formidable amount of numerical computations. To circumvent these difficulties, we developed a multiple point source method of computing $\mathbf{J}$. It has a potential to provide effective analysis and more accurate estimate of $\mathbf{J}$ near fishhook-shaped darts. Numerical experiments show that the MPSM is just fit for the study of EMD shocks.
\end{abstract}

Mathematics Subject Classification. 93A30, 32S05, 92C55, 33K28, 35M10, 35R30.

Received March 7, 2006.

\section{IntRoduction}

Electro-muscular disruption (EMD) devices such as TASER M26 and X26 are being used as a less-than-lethal weapon [27]. These devices generate a powerful electrical shock to completely override the central nervous system of a victim and directly control a large amount of skeletal muscles. It shoots a pair of fishhook-shaped darts attached to 15-feet wires toward an intended target. When the darts are inserted in the victims's skin or clothing, the device generates a debilitating electrical shock. Although these devices are intended to reduce the probability of fatality or permanent injury compared with other lethal weapons, there are ongoing controversial

Keywords and phrases. Electro-muscular disruption (EMD) device, electrical current density, Maxwell equations, non-smooth boundary, elliptic partial differential equations, corner singularity.

1 Department of Mathematics, Yonsei University, Korea. ezhyun@yonsei.ac.kr

2 Department of Mathematics, Yonsei University and National Institute for Mathematical Science, Korea. seoj@yonsei.ac.kr

3 College of Electronics and Information, Kyung Hee University, Korea. ejwoo@khu.ac.kr 


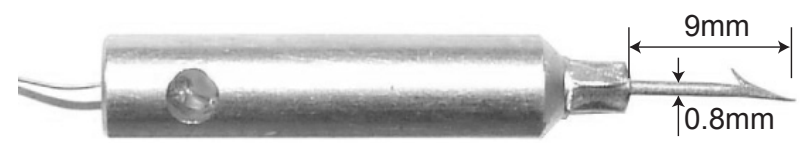

FIGURE 1. Fishhook-shaped dart used in typical EMD devices.

arguments over its safety as the number of death increases with more frequent uses of such EMD devices. See $[3,6,11,13,14,29]$. Numerous investigations on the implications of EMD shocks are, therefore, requested to create a guidance that helps device users to minimize the risk of injury or fatality of victims.

When the pair of darts strike a victim and an electrical circuit is formed, a current density $\mathbf{J}=\left(J_{x}, J_{y}, J_{z}\right)$ is established inside the body. The distribution of $\mathbf{J}$ is influenced by several factors such as the shape and location of darts, distance between darts, geometry of the body, and its conductivity distribution. The knowledge of $\mathbf{J}$ is necessary for the investigation of the risk of ventricular fibrilation (VF), seizure, skin burn, and so on.

In this paper, we suggest a basic study to undertake a three-dimensional numerical modelling of an EMD shock as a way to assess its biophysical implications. We setup a mathematical model of the EMD shock that can correctly simulate the real situation. Accurate computation of $\mathbf{J}$ near the dart is required to properly assess the risk of skin burn injury and VF in the worst case. In numerical computations of $\mathbf{J}$ using such a model, however, there are serious difficulties in dealing with the region of the fishhook-shaped dart since $\mathbf{J}$ is highly concentrated there. As shown in Figure 1, the dart is not axially symmetric and the inserted dart creates a three-dimensional computational domain with two different corners, tip of needle and tip of barb, where singularities of $|\mathbf{J}|$ occur. Therefore, dealing with these corners requires a very fine mesh to get enough numerical accuracy, and it may lead to a formidable amount of numerical computations and memory space. To circumvent these difficulties, we develop a multiple point source method (MPSM) of computing $\mathbf{J}$, that provides an analytical representation formula for the potential distribution near the fishhook-shaped dart. Eliminating the requirement of a very fine mesh, the MPSM seems to be an ideal method for us to model EMD shocks using a conventional PC.

In this paper, we try to explain the whole process of the current density imaging during EMD shocks; motivation, mathematical medelling, computational method, and a future study of experimental validation using animal subjects. We hope that the proposed computer model to simulate the effect of shocks help examine competing claims about the benefits and risks of TASERs $[3,13,27]$.

\section{Mathematical modelling of EMD shock}

\subsection{Problem definition}

In this section, we formulate a mathematical model of EMD shocks, bearing in mind that we are aiming to compute the current density distribution inside the body generated by a pair of inserted darts. We denote the three-dimensional domain of the subject as $\Omega$ with its boundary $\partial \Omega$. Let $\mathbf{r}=(x, y, z)$ denote a position vector. Since an EMD shock is induced by very short pulses of stimulating current, the subject $\Omega$ can be assumed to have an isotropic conductivity $\sigma$ that is a positive function defined in $\Omega$. The darts, denoted by $\mathcal{E}_{1}$ and $\mathcal{E}_{2}$, are fired from the device and have a fishhook-shaped geometry with $9 \mathrm{~mm}$ length and $0.8 \mathrm{~mm}$ diameter as shown in Figure 1.

An EMD device transmits electricity to the body $\Omega$ through the pair of darts, and it stimulates the subject with about 2 to $15 \mathrm{~A}$ body current and $50000 \mathrm{~V}$ peak voltage. We assume the injection current through the pair of darts is $I$ A. The current $I$ gives rise to a current density $\mathbf{J}=\left(J_{x}, J_{y}, J_{z}\right)$ inside the subject $\Omega_{s}:=\Omega \backslash \overline{\mathcal{E}_{1} \cup \mathcal{E}_{2}}$ 

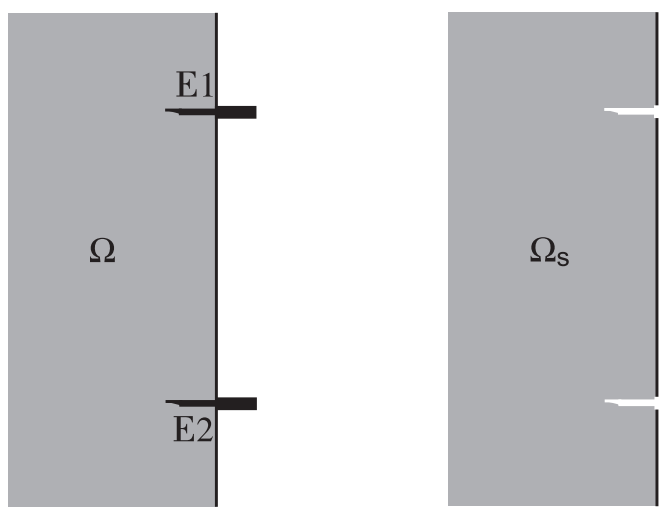

Figure 2. Simplified model of a computational domain with two darts.

depicted in Figure 2. For a given amount of injection current $I$, we have the following data along the darts:

$$
-\int_{\partial \mathcal{E}_{1}} \mathbf{J} \cdot \mathbf{n} \mathrm{d} s=I=\int_{\partial \mathcal{E}_{2}} \mathbf{J} \cdot \mathbf{n} \mathrm{d} s
$$

where $\partial \mathcal{E}_{j}$ is the boundary of the dart $\mathcal{E}_{j}$ intersecting $\Omega, \mathbf{n}$ the unit outward normal vector to the boundary $\partial \Omega_{s}$, and $\mathrm{d} s$ the surface area element. The current density $\mathbf{J}$ can be expressed as

$$
\mathbf{J}=\left(J_{x}, J_{y}, J_{z}\right)=-\sigma \nabla V
$$

where $V$ is the voltage inside the body $\Omega_{s}$. Since the dart is a very good conductor, $V$ can be assumed to be constant on each dart. Setting the voltage on the dart $\mathcal{E}_{2}$ to be zero, the voltage $V$ satisfies the following mixed boundary value problem with the elliptic PDE:

$$
\left\{\begin{array}{l}
\nabla \cdot(\sigma(\mathbf{r}) \nabla V(\mathbf{r}))=0 \text { in } \Omega_{s}:=\Omega \backslash \overline{\mathcal{E}_{1} \cup \mathcal{E}_{2}} \\
V=\text { a constant on } \partial \mathcal{E}_{1}, \quad V=0 \text { on } \partial \mathcal{E}_{2} \\
\sigma \frac{\partial V}{\partial \mathbf{n}}=0 \text { on } \partial \Omega \backslash \mathcal{E}_{1} \cup \mathcal{E}_{2} \\
\int_{\partial \mathcal{E}_{1}} \sigma \frac{\partial V}{\partial \mathbf{n}} \mathrm{d} s=I=-\int_{\partial \mathcal{E}_{2}} \sigma \frac{\partial V}{\partial \mathbf{n}} \mathrm{d} s
\end{array}\right.
$$

where $\frac{\partial V}{\partial \mathbf{n}}$ is the outward normal derivative of $V$. Here, $\partial \mathcal{E}_{j}$ is a Lipschitz boundary.

The model (2) is inconvenient as a computational model, so we need to modify it maintaining its structure. For a moment, we assume the voltage $V$ at the dart $\mathcal{E}_{1}$ is $\alpha$ and let $u=\frac{2 V}{\alpha}-1$. Then $u$ satisfies the following PDE:

$$
\left\{\begin{array}{l}
\nabla \cdot(\sigma \nabla u)=0 \text { in } \Omega_{s} \\
u=1 \text { on } \mathcal{E}_{1} \text { and } \\
\frac{\partial u}{\partial \mathbf{n}}=0 \text { on } \partial \Omega \backslash \overline{\mathcal{E}_{1} \cup \mathcal{E}_{2}} .
\end{array}\right.
$$

We need to determine $\alpha$ to compute $V$ directly from $u$ using the relation $V=\frac{\alpha}{2}(u+1)$. Taking normal derivative to $V=\frac{\alpha}{2}(u+1)$ and integrating it over $\partial \mathcal{E}_{1}$, we have $\int_{\partial \mathcal{E}_{1}} \sigma \frac{\partial V}{\partial \mathbf{n}} \mathrm{d} s=\frac{\alpha}{2} \int_{\partial \mathcal{E}_{1}} \sigma \frac{\partial u}{\partial \mathbf{n}} \mathrm{d} s$ and obtain the following linear relation:

$$
V(\mathbf{r})=\frac{\int_{\partial \mathcal{E}_{1}} \sigma \frac{\partial u}{\partial \mathbf{n}} \mathrm{d} s}{I}(u(\mathbf{r})+1), \quad \mathbf{r} \in \Omega_{s}
$$

Hence, it suffices to compute $u$ in the simplified model (3) for the computation of $\mathbf{J}=-\sigma \nabla V$ in the model (2). From this observation, it is easy to see the well-posedness of the model (2). 
For an accurate analysis of the distribution of $\mathbf{J}$ near the darts, we should carefully model the exact shape of the dart depicted in Figure 1. Assuming that the dart $\mathcal{E}_{1}$ is lying along the $z$-axis, we may express its geometry as follows:

$$
\begin{aligned}
\mathcal{E}_{1}:= & \left\{\mathbf{r} \in \mathbb{R}^{3}: \sqrt{x^{2}+y^{2}}<0.4,0<z \leq 6\right\} \\
& \cup\left\{\mathbf{r} \in \mathbb{R}^{3}: \sqrt{x^{2}+\left(y+\frac{2}{15}(z-6)\right)^{2}} \leq \frac{2(9-z)}{15}, 6 \leq z<9\right\} \\
& \cup\left\{\mathbf{r} \in \mathbb{R}^{3}: \sqrt{x^{2}+\left(y+\frac{6}{5}(z-6.4)\right)^{2}} \leq 0.2(z-5.4), 5.4 \leq z<6.4\right\} .
\end{aligned}
$$

\subsection{Concentration of $\mathbf{J}$ near fishhook-shaped dart}

In the region where darts are inserted, very high concentration of $\mathbf{J}$ is established. To see this in a quantitative way, let us compare $|\mathbf{J}|$ on $\mathcal{E}_{1}$ with $|\mathbf{J}|$ over the zero equipotential surface $\Gamma_{0}:=\{\mathbf{r} \in \Omega: u(\mathbf{r})=0\}$. We can view $\Gamma_{0}$ roughly as a cross-sectional surface lying somewhere between two darts since $\Gamma_{0}$ separates the body $\Omega_{s}$ into two parts $\Omega_{s}^{+}:=\left\{\mathbf{r} \in \Omega_{s}: u(\mathbf{r})>0\right\}$ and $\Omega_{s}^{-}=\Omega_{s} \backslash \Omega_{s}^{+}$. According to the divergence theorem, we have

$$
0=\int_{\Omega_{s}^{+}} \nabla \cdot(\sigma \nabla u) \mathrm{d} \mathbf{r}=\int_{\partial \Omega_{s}^{+}} \sigma \frac{\partial u}{\partial \mathbf{n}} \mathrm{d} S=\int_{\partial \mathcal{E}_{1}} \sigma \frac{\partial u}{\partial \mathbf{n}} \mathrm{d} S+\int_{\Gamma_{0}} \sigma \frac{\partial u}{\partial \mathbf{n}} \mathrm{d} S
$$

where $\Omega_{s}^{+}:=\left\{\mathbf{r} \in \Omega_{s}: u(\mathbf{r})>0\right\}$. Since the normal vector on the equipotential surface is $\mathbf{n}=\frac{\nabla u}{|\nabla u|}$ on $\partial \mathcal{E}_{1}$ and $\mathbf{n}=\frac{\nabla u}{|\nabla u|}$ on $\Gamma_{0}$, the above identity becomes

$$
\int_{\partial \mathcal{E}_{1}}|\mathbf{J}| \mathrm{d} S=c \int_{\partial \mathcal{E}_{1}}|\sigma \nabla u| \mathrm{d} S=c \int_{\Gamma_{0}}|\sigma \nabla u| \mathrm{d} S=\int_{\Gamma_{0}}|\mathbf{J}| \mathrm{d} S
$$

where $c=\frac{1}{I} \int_{\partial \mathcal{E}_{1}} \sigma \frac{\partial u}{\partial \mathbf{n}} \mathrm{d} s$ is the scaling constant. Note that the surface area of the dart is approximately $0.8 \pi \times 9 \mathrm{~mm}^{2}$ and the surface area of a cross-section of a normal adult body would be bigger than $200 \times 300 \mathrm{~mm}^{2}$. Hence, we may conclude that

$$
\text { [average } \left.|\mathbf{J}| \text { on } \partial \mathcal{E}_{1}\right] \geq 2000 \times\left[\text { average }|\mathbf{J}| \text { on the cross-section } \Gamma_{0}\right]
$$

For the risk characterization, therefore, we must focus on $\mathbf{J}$ near the darts, while we may disregard $\mathbf{J}$ at some distance from the darts. In particular, we should carefully investigate the magnitude of $\mathbf{J}$ near the dart $\mathcal{E}_{1}$ where it is placed with the shortest distance to the heart as the worst case.

\section{Multiple point source method (MPSM)}

\subsection{Homogeneous half-space model}

In the model (3), we need to develop a computational method providing reliable estimates of $\mathbf{J}$ near darts with various positions. The computational domain has two corners at the dart, tip of needle and tip of barb, where singularities of $\mathbf{J}$ occur. Since the tip and barb of the dart of this TASER model do not have any radial symmetry, any asymptotic formula of the gradient of the solution in this TASER model is not available. Conventional FEM requires a very fine mesh near the fishhook-shaped corners to get enough numerical accuracy and it leads to a large-scale computation. The proposed MPSM effectively deals with complicated singularities at very sharp small corners.

To explain the MPSM, we begin with considering the case where the subject is the upper half-space $\mathbb{R}_{+}^{3}=$ $\{\mathbf{r}=(x, y, z): z>0\}$ and has a homogeneous conductivity. Let $u_{1}$ be the solution of (3) with $\Omega$ replaced by 
$\mathbb{R}_{+}^{3}$ and $\lim _{\mathbf{r} \rightarrow \infty} u_{1}(\mathbf{r})=0$. Then we have

$$
\left\{\begin{array}{l}
\nabla^{2} u_{1}=0 \text { in } \mathbb{R}_{+}^{3} \backslash \overline{\mathcal{E}_{1} \cup \mathcal{E}_{2}} \\
u_{1}=1 \text { on } \partial \mathcal{E}_{1} \text { and } \\
\frac{\partial u_{1}}{\partial \mathbf{n}}=0 \text { on } \partial \mathbb{R}_{+}^{3} \backslash \overline{\mathcal{E}_{1} \cup \mathcal{E}_{2}} \\
\lim _{\mathbf{r} \rightarrow \infty} u_{1}(\mathbf{r})=0
\end{array}\right.
$$

where $\nabla^{2}=\nabla \cdot \nabla$ denotes the Laplacian.

The basic idea of the MPSM is to view the dart as a sum of a sequence of point sources lying inside the dart and their image sources with respect to the $z$-plane. Let $\mathbf{a}_{0}, \mathbf{a}_{1}, \ldots, \mathbf{a}_{M_{1}}$ be a sequence of points lying inside the dart $\mathcal{E}_{1}$ and let $\mathbf{a}_{j}^{-}$be the reflected point of $\mathbf{a}_{j}$ to the $z$-plane. Similarly, let $\mathbf{b}_{0}, \ldots, \mathbf{b}_{M_{2}} \in \mathcal{E}_{2}$ with their reflected points $\mathbf{b}_{j}^{-}$. Now we consider the multiple-source function

$$
W(\mathbf{r})=\sum_{j=0}^{M_{1}} q_{j}^{1}\left(\frac{1}{\left|\mathbf{r}-\mathbf{a}_{j}\right|}+\frac{1}{\left|\mathbf{r}-\mathbf{a}_{j}^{-}\right|}\right)-\sum_{j=0}^{M_{2}} q_{j}^{2}\left(\frac{1}{\left|\mathbf{r}-\mathbf{b}_{j}\right|}+\frac{1}{\left|\mathbf{r}-\mathbf{b}_{j}^{-}\right|}\right)
$$

where $q_{j}^{1}$ and $q_{j}^{2}$ are positive point charges. This $W$ satisfies

$$
\nabla^{2} W(\mathbf{r})=0 \quad \text { in } \quad \Omega_{s}=R_{+}^{3} \backslash\left(\mathcal{E}_{1} \cup \mathcal{E}_{2}\right) \quad \text { and } \quad \lim _{|\mathbf{r}| \rightarrow \infty} W(\mathbf{r})=0
$$

Due to the presence of the image sources at $\mathbf{a}_{j}^{-}$and $\mathbf{b}_{j}^{-}$, we have

$$
\frac{\partial}{\partial z} W(\mathbf{r})=0 \quad \text { on the } z \text {-plane. }
$$

Hence, $W$ satisfies all the conditions of the model $(3)$ but the boundary conditions on the darts. If we could approximate $W \approx 1$ on $\mathcal{E}_{1}$ and $W \approx-1$ on $\mathcal{E}_{2}$, then $W \approx u_{1}$ in the entire domain $\Omega_{s}$ according to the standard maximum principle.

In order for $W$ to be a good approximation of $u_{1}$, we need to find appropriate source points and point charges such that $W \approx 1$ on $\mathcal{E}_{1}$ and $W \approx-1$ on $\mathcal{E}_{2}$. To be precise, we need to find the vectors $\mathbf{a}_{0}, \ldots, \mathbf{a}_{M_{1}} \in \mathcal{E}_{1}$, $\mathbf{b}_{0}, \ldots, \mathbf{b}_{M_{2}} \in \mathcal{E}_{2}$, and positive quantities $q_{0}^{1}, \ldots, q_{M_{1}}^{1}, q_{0}^{2}, \ldots, q_{M_{1}}^{2}$ which minimize the following functional

$$
\begin{aligned}
\int_{\partial \mathcal{E}_{1}} \mid 1- & \left.W(\mathbf{r})\right|^{2} \mathrm{~d} s+\int_{\partial \mathcal{E}_{2}}|1+W(\mathbf{r})|^{2} \mathrm{~d} s \\
= & \int_{\partial \mathcal{E}_{1}}\left|1-\sum_{j=0}^{M_{1}} q_{j}^{1} F_{j}^{1}(\mathbf{r})+\sum_{j=0}^{M_{2}} q_{j}^{2} F_{j}^{2}(\mathbf{r})\right|^{2} \mathrm{~d} s \\
& \quad+\int_{\partial \mathcal{E}_{2}}\left|1+\sum_{j=0}^{M_{1}} q_{j}^{1} F_{j}^{1}(\mathbf{r})-\sum_{j=0}^{M_{2}} q_{j}^{2} F_{j}^{2}(\mathbf{r})\right|^{2} \mathrm{~d} s
\end{aligned}
$$

where

$$
F_{j}^{1}(\mathbf{r}):=\frac{1}{\left|\mathbf{r}-\mathbf{a}_{j}\right|}+\frac{1}{\left|\mathbf{r}-\mathbf{a}_{j}^{+}\right|} \quad \text { and } \quad F_{j}^{2}(\mathbf{r}):=\frac{1}{\left|\mathbf{r}-\mathbf{b}_{j}\right|}+\frac{1}{\left|\mathbf{r}-\mathbf{b}_{j}^{+}\right|}
$$

To simplify the minimization problem, we may choose $\mathbf{a}_{j}$ and $\mathbf{b}_{j}$ by considering the distribution of the electric potential due to the special geometry of the fishhook-shaped dart $\mathcal{E}_{1}$. For example, one can choose

$$
\mathbf{a}_{j}= \begin{cases}\left(0,-0.4+\frac{2}{5}(1.4)^{j-8}, 9-3(1.4)^{j-8}\right), & 0 \leq j \leq 7 \\ \left(0,0, \frac{5(j-8)}{8}\right), & 8 \leq j \leq 15 \\ \left(0,0,5+\frac{j-16}{8}\right), & 16 \leq j \leq 23 \\ \left(0, \frac{6(j-24)}{40}, 6.4-\frac{j-24}{8}\right), & 24 \leq j \leq 33\end{cases}
$$


Some theoretical insight about corner singularity is generally required for an appropriate choice of such source points.

With the given $\mathbf{a}_{0}, \ldots, \mathbf{a}_{M_{1}}, \mathbf{b}_{0}, \ldots, \mathbf{b}_{M_{2}}$, the minimization problem in (7) can be reduced to find a vector $\mathbf{q}=\left(q_{0}^{1}, \ldots, q_{M_{1}}^{1}, q_{0}^{2}, \ldots, q_{M_{2}}^{2}\right)$ minimizing the following functional:

$$
\Phi(\mathbf{q})=\int_{\partial \mathcal{E}_{1}}|1-\mathbf{q} \cdot \mathbb{F}(\mathbf{r})|^{2} \mathrm{~d} s+\int_{\partial \mathcal{E}_{2}}|1+\mathbf{q} \cdot \mathbb{F}(\mathbf{r})|^{2} \mathrm{~d} s
$$

where

$$
\mathbb{F}(\mathbf{r})=\left(F_{0}^{1}(\mathbf{r}), \ldots, F_{M_{1}}^{1}(\mathbf{r}),-F_{0}^{2}(\mathbf{r}), \ldots,-F_{M_{2}}^{2}(\mathbf{r})\right) .
$$

To discretize (8), we choose a sequence of points on the boundary of darts such as $\mathbf{r}_{1}^{1}, \ldots, \mathbf{r}_{N_{1}}^{1} \in \partial \mathcal{E}_{1}$ and $\mathbf{r}_{1}^{2}, \ldots, \mathbf{r}_{N_{2}}^{2} \in \partial \mathcal{E}_{2}$. Then, this minimization problem can be simplified to find $\mathbf{q}$ that minimizes the following sum:

$$
\tilde{\Phi}(\mathbf{q})=\sum_{k=0}^{N_{1}}\left|1-\mathbf{q} \cdot \mathbb{F}\left(\mathbf{r}_{k}^{1}\right)\right|^{2}+\sum_{k=0}^{N_{2}}\left|1+\mathbf{q} \cdot \mathbb{F}\left(\mathbf{r}_{k}^{2}\right)\right|^{2} .
$$

For readers who try to test the MPSM, we provide an example of $\mathbf{r}_{j}^{1}$ :

$$
\mathbf{r}_{j}^{1}=\left\{\begin{array}{ll}
\left(0,-0.4,9-3(1.4)^{j-N}\right), & j=0, \ldots, N-1 \\
\left(0,0.4, \frac{5(j-N)}{N}\right), & N \leq j \leq 2 N-1 \\
\left.0,0.4,5+\frac{(j-2 N)}{N}\right), & 2 N \leq j \leq 3 N-1 \\
\left.0,0.4+\frac{6(j-3 N)}{5 N}, 6.4-\frac{j-3 N}{N}\right) & 3 N \leq j \leq 4 N-1 \\
\left.0,0.4, \frac{6(j-4 N)}{N}\right), & 4 N \leq j \leq 5 N-1
\end{array} \text { and } N=8 .\right.
$$

If $\mathbf{q}^{*}$ is a minimizer of the functional $\tilde{\Phi}(\mathbf{q})$ in (9), then the solution $u_{1}$ can be approximated as

$$
u_{1}(\mathbf{r}) \approx \mathbf{q}^{*} \cdot \mathbb{F}(\mathbf{r}), \quad \mathbf{r} \in \mathbb{R}_{+}^{3} \backslash \overline{\mathcal{E}_{1} \cup \mathcal{E}_{2}} .
$$

\subsubsection{Remark on MPSM}

The MPSM is very flexible and it can be viewed as a dart-drawing process by appropriately arranging point sources at $\mathbf{a}_{j}$. The choice of the number of poles should depend on the global geometry of the dart and the singular structure of the gradient of the solution. Some knowledge about corner singularities in elliptic PDE and layer potential theory may help determine a desirable arrangement of $\mathbf{a}_{j}$ by looking at the geometry of the dart, or one can learn it heuristically by using a computer program. In particular, since the barb of the dart is very thin, we need to arrange poles densely, and position of them should be located a little bit on the upper part of the center line of the barb due to the influence of poles of the main cylindrical part of the dart from which the barb branches out.

Let us state a simple theoretical lemma that is related to the MPSM.

Lemma 3.1. Let $u_{1}$ be the solution of (5). There exist $q^{1} \in L^{2}\left(\partial \mathcal{E}_{1}^{*}\right)$ and $q^{2} \in L^{2}\left(\partial \mathcal{E}_{2}^{*}\right)$ such that $q^{1}$ and $q^{2}$ are symmetric with respect to $z$-axis and

$$
u_{1}(\mathbf{r})=\mathcal{S}_{\partial \mathcal{E}_{1}^{*}} q^{1}(\mathbf{r})-\mathcal{S}_{\partial \mathcal{E}_{2}^{*}} q^{2}(\mathbf{r}) \quad \text { in } \mathbb{R}_{+}^{3} \backslash \overline{\mathcal{E}_{1} \cup \mathcal{E}_{2}}
$$

where $\mathcal{E}_{j}^{*}$ is the interior domain of $\overline{\mathcal{E}_{j} \cup\left\{\mathbf{r} \in \mathbb{R}^{3}:(x, y,-z) \in \mathcal{E}_{j}\right\}}, j=1,2$ and $\mathcal{S}_{\partial \mathcal{E}_{j}^{*}} q^{j}$ is the single layer potential $\mathcal{S}_{\partial \mathcal{E}_{j}^{*}} q^{j}(\mathbf{r}):=\int_{\partial \mathcal{E}_{j}^{*}} \frac{-1}{4 \pi\left|\mathbf{r}-\mathbf{r}^{\prime}\right|} q^{j}\left(\mathbf{r}^{\prime}\right) \mathrm{d} s_{\mathbf{r}^{\prime}}$. 

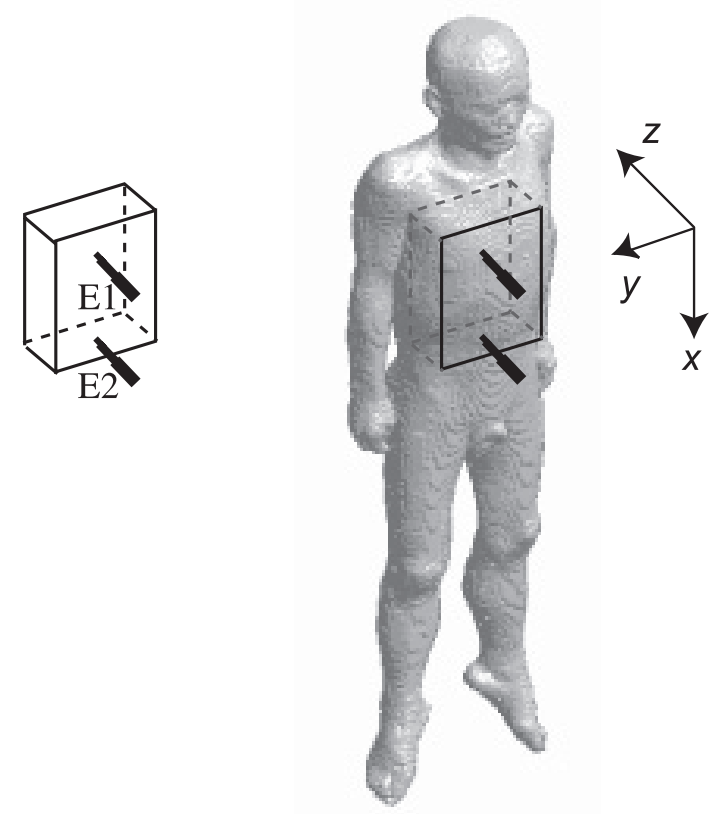

(a)

(b)

Figure 3. (a) Hexahedral domain and (b) human body model.

Proof. We will give a brief proof. We extend $u_{1}$ into the lower half-space by even reflection, so the extended function $u_{1}^{*}$ is defined by $u_{1}^{*}(x, y,-z)=u_{1}(x, y, z)$ for $(x, y, z) \in \mathbb{R}_{+}^{3} \backslash \overline{\mathcal{E}_{1} \cup \mathcal{E}_{2}}$. Then $u_{1}^{*}$ satisfies the Dirichlet problem

$$
\nabla^{2} u_{1}^{*}=0 \quad \text { in } \quad \mathbb{R}^{3} \backslash \overline{\mathcal{E}_{1}^{*} \cup \mathcal{E}_{2}^{*},} \quad u_{1}^{*}=1 \quad \text { on } \partial \mathcal{E}_{1}^{*}, \quad u_{1}^{*}=-1 \quad \text { on } \partial \mathcal{E}_{2}^{*} .
$$

Then the proof follows from the invertibility of single layer potential on Lipschitz domain [28].

Since the dart $\mathcal{E}_{j}$ is very thin and sharp, it is like a curve. Hence, the above lemma can be modified as follows. For appropriately given curves $\gamma_{j}$ contained in $\mathcal{E}_{j}^{*}$, there exist charges $q^{j} \in L^{2}\left(\gamma_{j}\right)$ such that

$$
\int_{\gamma_{1}} \frac{1}{4 \pi\left|\mathbf{r}-\mathbf{r}^{\prime}\right|} q^{1}\left(\mathbf{r}^{\prime}\right) \mathrm{d} l_{\mathbf{r}^{\prime}}-\int_{\gamma_{2}} \frac{1}{4 \pi\left|\mathbf{r}-\mathbf{r}^{\prime}\right|} q^{2}\left(\mathbf{r}^{\prime}\right) \mathrm{d} l_{\mathbf{r}^{\prime}} \approx\left\{\begin{array}{cll}
1 & \text { on } & \partial \mathcal{E}_{1} \\
-1 & \text { on } & \partial \mathcal{E}_{2}
\end{array}\right.
$$

where $\mathrm{d} l$ is the length element. The MPSM can be viewed as a discretized version of the above approximation.

\subsection{Computation of $\mathbf{J}$ in bounded domain and finite geometry effect}

\subsubsection{Hexahedral domain with homogeneous conductivity}

Now, we consider the solution $u$ of (3) in a bounded domain $\Omega_{s}$ with a homogeneous conductivity. We begin with the case where $\Omega$ is a hexahedral domain with $\sigma=1$ as shown in Figure 3a. To be precise, let

$$
\Omega:=\{\mathbf{r}=(x, y, z):-100 \mathrm{~mm}<x<400 \mathrm{~mm},|y|<100 \mathrm{~mm}, 0<z<150 \mathrm{~mm}\} .
$$

Assume that the dart $\mathcal{E}_{1}$ strikes at the origin. The other dart $\mathcal{E}_{2}$ strikes $L \mathrm{~mm}$ below $\mathcal{E}_{1}$ and $100 \mathrm{~mm}<L<$ $300 \mathrm{~mm}$, that is, $\mathcal{E}_{2}=\left\{\mathbf{r}+(L, 0,0): \mathbf{r} \in \mathcal{E}_{1}\right\}$. 
For the computation of $u$, we make a good use of the approximation $u_{1}(\mathbf{r}) \approx \mathbf{q}^{*} \cdot \mathbb{F}(\mathbf{r})$ in (10). We decompose the solution $u$ of (3) into

$$
u(\mathbf{r})=u_{1}(\mathbf{r})+\phi(\mathbf{r}), \quad \mathbf{r} \in \Omega_{s} .
$$

Then, $\phi$ satisfies the following mixed boundary value problem:

$$
\left\{\begin{array}{c}
\nabla^{2} \phi=0 \text { in } \Omega_{s} \\
\phi=0 \text { on } \partial \mathcal{E}_{1} \cup \partial \mathcal{E}_{2} \\
\frac{\partial \phi}{\partial \mathbf{n}}=-\frac{\partial u_{1}}{\partial \mathbf{n}} \quad \text { on } \partial \Omega \backslash \overline{\mathcal{E}_{1} \cup \mathcal{E}_{2}}
\end{array}\right.
$$

The quantity $\nabla \phi$ can be viewed as a finite geometry effect. Due to the presence of the sharp darts $\mathcal{E}_{1}$ and $\mathcal{E}_{2}$ in $\Omega$, the computation of $\phi$ is again a formidable job. In order to avoid this, we decompose $\phi$ again into two parts:

$$
\phi(\mathbf{r})=\phi_{1}(\mathbf{r})+\phi_{2}(\mathbf{r})
$$

where $\phi_{1}$ is the solution of the following standard Neumann boundary problem:

$$
\left\{\begin{array}{l}
\nabla^{2} \phi_{1}=0 \quad \text { in } \Omega \\
\mathbf{n} \cdot \nabla \phi_{2}=-\mathbf{n} \cdot \nabla u_{1} \quad \text { on } \partial \Omega, \quad \int_{\partial \Omega} \phi_{1}=0
\end{array}\right.
$$

and $\phi_{2}$ satisfies

$$
\left\{\begin{array}{c}
\nabla^{2} \phi_{2}=0 \text { in } \Omega_{s} \\
\phi_{2}=-\phi_{1} \quad \text { on } \partial \mathcal{E}_{1} \cup \partial \mathcal{E}_{2} \\
\mathbf{n} \cdot \nabla \phi_{2}=0
\end{array}\right.
$$

Note that the computation of $\phi_{1}$ is easy via any standard numerical method without using a fine mesh.

Next, we consider the second term $\phi_{2}$. We conjecture that the ratio $\frac{\left|\nabla \phi_{2}\right|}{|\nabla u|}$ is very small in $\Omega_{s} \cap B$ where $B$ is the ball with $30 \mathrm{~mm}$ radius and centered at the origin. Then we can neglect the second term $\phi_{2}$ in $\Omega_{s} \cap B$, the region of interest. Although this conjecture seems to be obvious, we are not able to rigorously prove it. Indeed, even the clear fact that $|\nabla u|>0$ in $\Omega_{s} \cap B$ is very difficult to be proved in a three-dimensional case, while there are some related two-dimensional results in $[1,2,23]$. We think that these theoretical issues should be addressed in future studies, although those are obvious from physical insight.

Without a rigorous proof, we try to explain a reason why $\frac{\left|\nabla \phi_{2}\right|}{|\nabla u|}$ is very small in $\Omega_{s} \cap B$. According to the expression of $u_{1}$ in (10), the quantity $\left|\nabla u_{1}(\mathbf{r})\right|$ decays with a rate of the square of the distance from the dart. The solution $\phi_{1}$ of (14) has the Neumann boundary condition $\frac{\partial \phi_{1}}{\partial \mathbf{n}}=-\frac{\partial u_{1}}{\partial \mathbf{n}}$ which is very small on $\partial \Omega$ due to the decaying property of $u_{1}$. Hence, the contribution of $\nabla \phi_{1}$ to $\nabla u$ near the dart would be very small. We performed numerical computations of $\phi_{1}$ with various $100 \mathrm{~mm} \leq L \leq 300 \mathrm{~mm}$ and the results showed that $\left|\nabla \phi_{1}\right| \approx 0$ near $\mathcal{E}_{1}$ and the difference of $\phi_{1}$ between $\mathcal{E}_{1}$ and $\mathcal{E}_{2}$ are less than 0.01 . These crude estimates yield that $\phi_{1} \approx c_{1}$ on $\mathcal{E}_{1}$ and $\phi_{1} \approx c_{2}$ on $\mathcal{E}_{2}$ and $0<\left|c_{1}-c_{2}\right|<0.01$. Since $\phi_{2}$ satisfies the same boundary value problem with $u$ in (3) except some difference on $\partial \mathcal{E}_{1} \cup \partial \mathcal{E}_{2}, u$ can be approximated by

$$
u(\mathbf{r}) \approx \frac{2}{c_{1}-c_{2}} \phi_{2}(\mathbf{r})+1-\frac{2 c_{1}}{c_{1}-c_{2}} .
$$

Hence, $\frac{\left|\nabla \phi_{2}\right|}{|\nabla u|} \approx \frac{\left|c_{1}-c_{2}\right|}{2} \leq 0.005$ in the region $\Omega_{s} \cap B$. We again stress that this estimate is not proven rigorously, so it is an open problem.

The above estimate means that $\phi_{2}$ has less than $1 \%$ effect on $\mathbf{J}$ in the region of interest. Hence, we can approximate

$$
\nabla u \approx \nabla u_{1}+\nabla \phi_{1} \approx \nabla\left(\mathbf{q}^{*} \cdot \mathbb{F}(\mathbf{r})\right)+\nabla \phi_{1}
$$

The major advantage of this approximation is that the two terms of the right side of (15) are computable using a PC. Various numerical simulations show that $u_{1}+\phi_{1}$ satisfies (3) with the Dirichlet conditions $u_{1}+\phi_{1} \approx 1$ on $\mathcal{E}_{1}$ and $u_{1}+\phi_{1} \approx-1$ on $\mathcal{E}_{2}$. Hence, $u_{1}+\phi_{1}$ can be viewed as an accurate numerical solution of (3). 


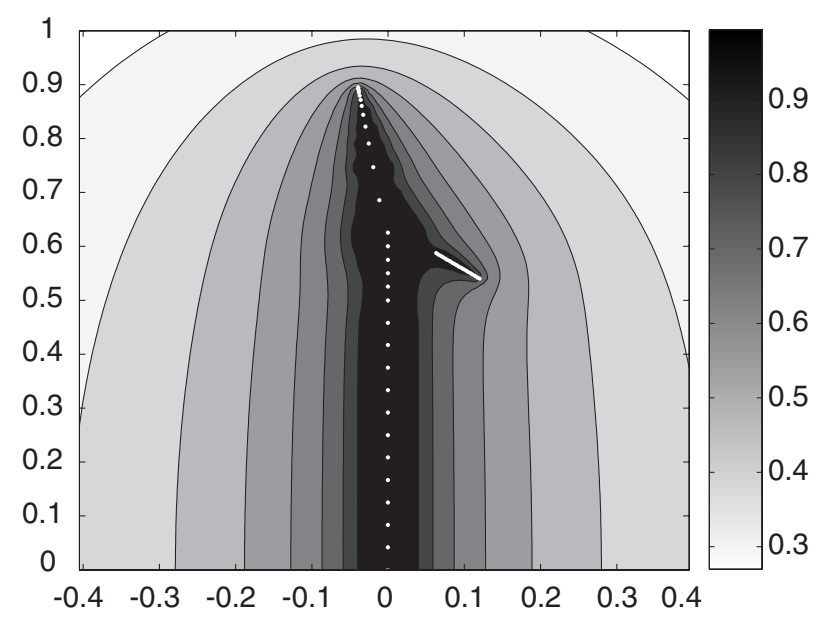

FIGURE 4. Modelling of the fishhook-shaped dart using the MPSM. White dots inside the dart are source points.

\subsubsection{Human body model with homogeneous conductivity}

Now, let $\Omega$ be the human body model shown in Figure $3 \mathrm{~b}$ and let $\Omega_{*}$ be the hexahedral domain in (11). The three-dimensional model in Figure 3b was generated by using the Visible Korean Human data set [20]. We still assume that the conductivity $\sigma$ is homogeneous. When the origin is positioned at the pit of the stomach, most adult human bodies can contain the hexahedral domain $\Omega_{*}$ with an appropriate coordinate system. Then we are considering a worst case where the dart $\mathcal{E}_{1}$ strikes at the pit of the stomach. For simplicity, we assume $\Omega_{*} \cap\{z=0\} \subset \Omega \cap\{z=0\}$, which is reasonable. Since $\partial \Omega \cap\{z=0\}$ is outside the hexahedron $\Omega_{*}$, the boundary effect to $\mathbf{J}$ near the dart is smaller than that is in the case of $\Omega_{*}$ only. For the human body model $\Omega$ under the assumption of a homogeneous conductivity, the approximation (15) also holds.

\section{NUMERICAL EXPERIMENTS}

\subsection{Modelling of fishhook-shaped dart using MPSM}

The MPSM provides an efficient way to model the fishhook-shaped dart by arranging point sources inside the dart. A sequence of source points $\mathbf{a}_{j}$ and $\mathbf{a}_{j}^{-}$were chosen as in 3.1 by considering the voltage distribution due to the special geometry of the dart. Now, the shape modelling problem reduces to find appropriate point charges to enforce the boundary condition on the dart. In order to solve this, we employed the discrete minimization using boundary points of the dart, $\mathbf{r}_{i}$ given in 3.1. Then we approximated multiple-source function $W$ and Figure 4 shows the contour plot of the obtained voltage distribution. Equipotential surface of $V=1$ denoted as black color matches well with the fishhook-shaped dart.

\subsection{Distribution of voltage}

Figure $5 \mathrm{a}-\mathrm{c}$ show distributions of voltage from the homogeneous half-space model with increasing field-ofview. Figure $6 \mathrm{a}-\mathrm{c}$ are the corresponding voltage distributions computed from the hexagonal model. Using the homogeneous human body model, voltage distributions were almost identical to those from the hexagonal model. As expected, the voltage distribution near each dart in the bounded domain changes little compared with that in the half-space. In the region far away from both darts, we can observe differences in voltage distributions. However, the gradients of voltage there are small. 


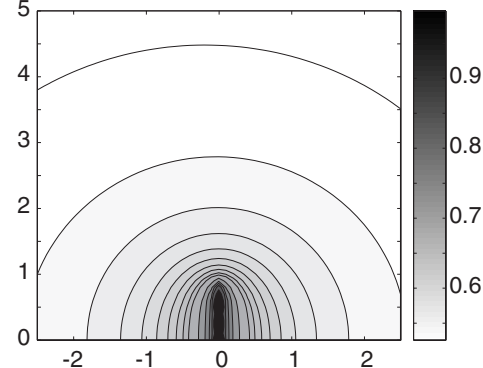

(a)

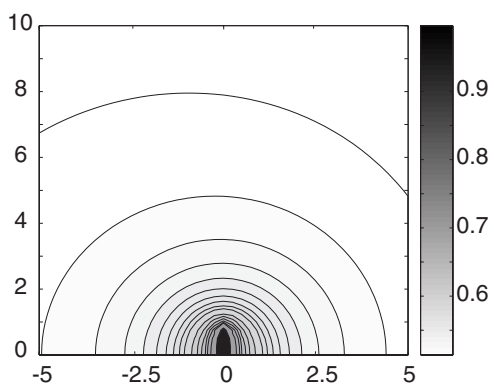

(b)

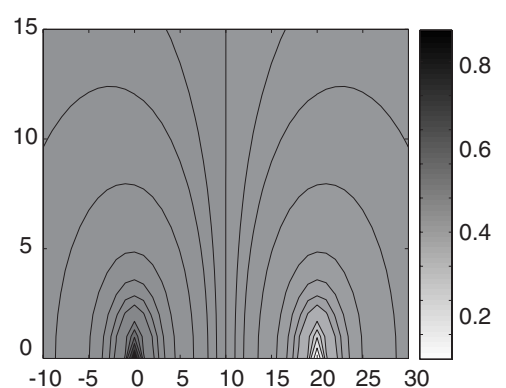

(c)

FiguRE 5. Distribution of voltage from the half-space model with different field-of-view: (a) $5 \times$ $5 \mathrm{~cm}^{2}$, (b) $10 \times 10 \mathrm{~cm}^{2}$ and (c) $40 \times 15 \mathrm{~cm}^{2}$.

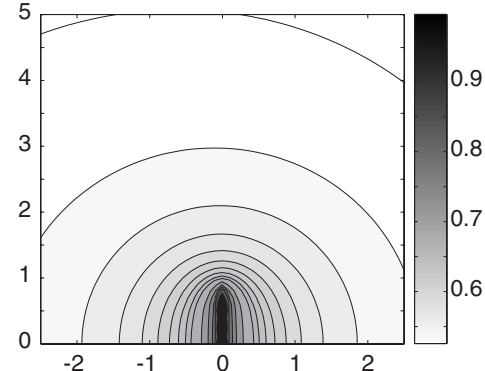

(a)

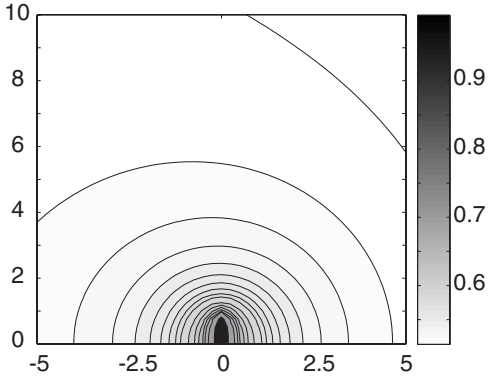

(b)

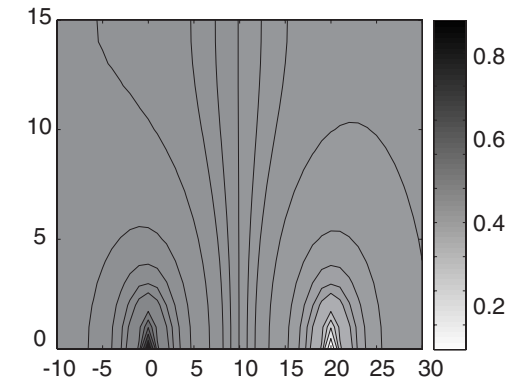

(c)

FIGURE 6. Distribution of voltage from the hexagonal model with different field-of-view: (a) $5 \times$ $5 \mathrm{~cm}^{2}$, (b) $10 \times 10 \mathrm{~cm}^{2}$ and (c) $40 \times 15 \mathrm{~cm}^{2}$.

\subsection{Distribution of magnitude of current density}

Figure $7 \mathrm{a}-\mathrm{c}$ are distributions of magnitude of current density from the homogeneous half-space model with increasing field-of-view. Figure $8 \mathrm{a}-\mathrm{c}$ are the corresponding distributions of magnitude of current density from the hexagonal model. The homogeneous human body model produced almost identical results as in Figure 8a-c. Figure 9 shows how the magnitude of current density decays as we move along the straight line starting from the tip of the dart. In all three homogeneous models, the plots are almost identical within $13 \mathrm{~cm}$ distance from the tip of the dart.

\section{Discussions ON REALISTIC HUMAN BODY MODEL AND ANIMAL EXPERIMENTS}

The conductivity distribution of a realistic human body model should not be homogeneous since different tissues have different conductivity values. For a realistic human body model, therefore, we need a threedimensional human anatomy data set with knowledge of conductivity distribution. As illustrated in Figure 3b, we are using the Visible Korean Human data set that includes segmented cross-sectional images of a Korean adult male cadaver [20]. We are currently conducting numerical simulations using this realistic human body model by assigning appropriate conductivity values to the segmented internal regions. Though this ongoing research requires a great deal of efforts and time, we speculate that the three-dimensional realistic human body model together with the MPSM suggested in this paper will be able to provide the most reliable numerical simulation results of EMD shocks. Especially we should focus on properly incorporating an inhomogeneous 


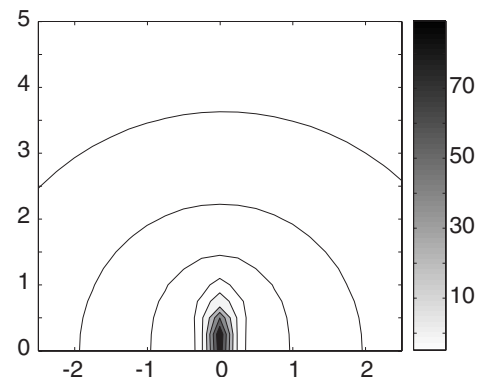

(a)

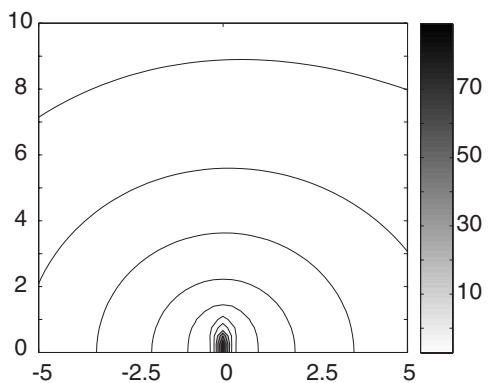

(b)

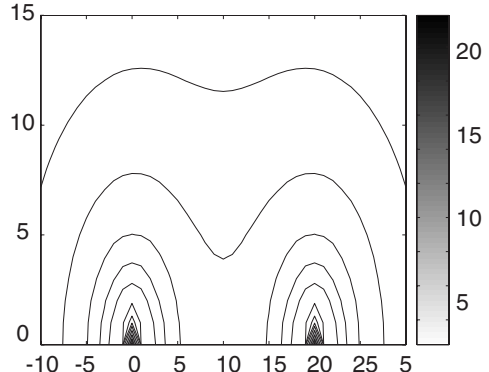

(c)

FIGURE 7. Distribution of magnitude of current density from the half-space model with different field-of-view: (a) $5 \times 5 \mathrm{~cm}^{2}$, (b) $10 \times 10 \mathrm{~cm}^{2}$ and (c) $40 \times 15 \mathrm{~cm}^{2}$.

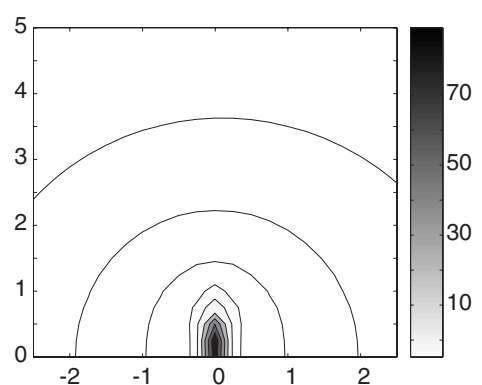

(a)

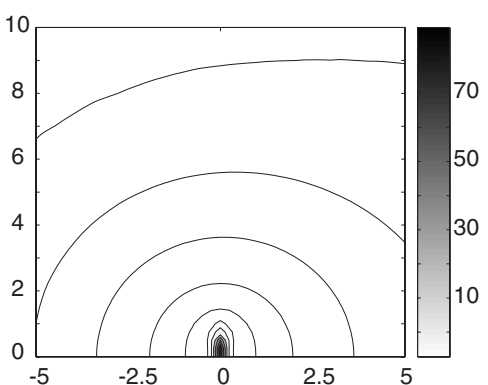

(b)

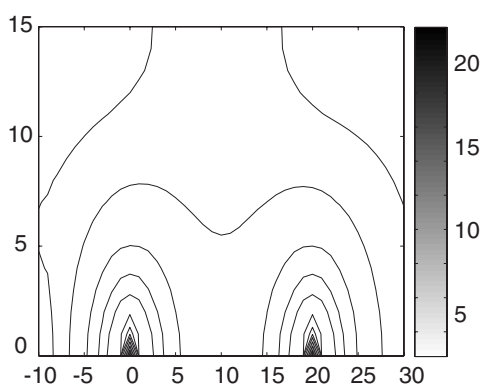

(c)

FIGURE 8. Distribution of magnitude of current density from the hexagonal model with different field-of-view: (a) $5 \times 5 \mathrm{~cm}^{2}$, (b) $10 \times 10 \mathrm{~cm}^{2}$ and (c) $40 \times 15 \mathrm{~cm}^{2}$.

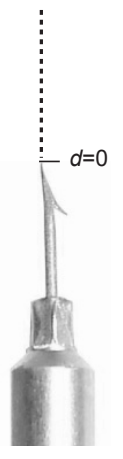

(a)

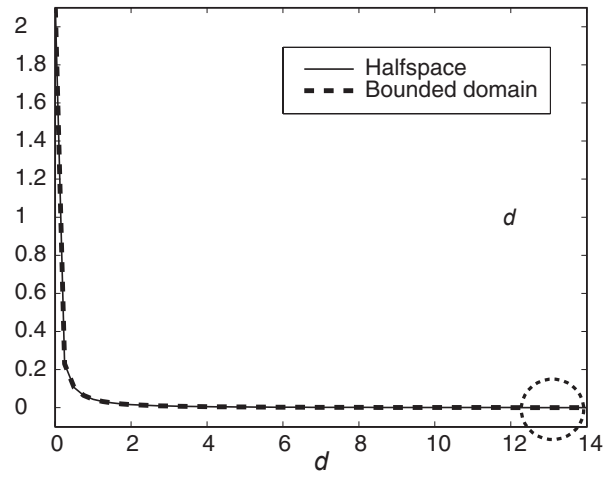

(b)

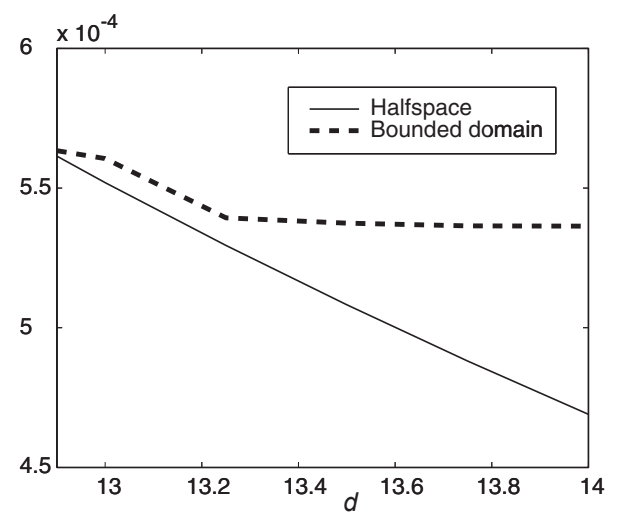

(c)

Figure 9. (a) We are interested in the magnitude of current density along the dotted line where $d$ is the distance from the tip of the dart. (b) Decay of magnitude of current density where $x$-axis is the distance $d$ from the tip of the dart. (c) is the magnified plot of the portion circled in (b). 
realistic conductivity distribution into the human body model since it is highly possible that such a conductivity distribution will alter voltage and current density distributions even in a region near the fishhook-shaped dart.

Using the realistic human body model, we apply the approximation (10) inside a small region of pixels containing the dart to handle singularities at the sharp corners. For example,

$$
\nabla u(r) \approx \nabla u_{1}(\mathbf{r})+\nabla \phi_{1}(\mathbf{r}) \quad \text { in } \quad \Omega_{s} \cap\left(D_{1} \cup D_{2}\right)
$$

where $D_{1}=\{\mathbf{r}:|x|<\alpha,|y|<\alpha, 0<z<\alpha+10\}, D_{2}=\left\{\mathbf{r}+(L, 0,0): \mathbf{r} \in D_{1}\right\}$, and $\alpha$ is a small number depending on a given pixel size of the segmented anatomy image data. For the computation of $u$ in $\Omega \backslash \overline{D_{1} \cup D_{2}}$, we may solve the following problem:

$$
\left\{\begin{array}{l}
\nabla \cdot(\sigma \nabla u)=0 \text { in } \Omega \backslash \overline{D_{1} \cup D_{2}} \\
\sigma \frac{\partial u}{\frac{\partial u}{\mathbf{n}}}=\sigma \frac{\partial u_{1}}{\partial \mathbf{n}}+\sigma \frac{\partial \phi_{1}}{\partial \mathbf{n}} \quad \text { on } \partial\left(D_{1} \cup D_{2}\right) \cap \Omega \\
\sigma \frac{\partial u}{\partial \mathbf{n}}=0 \text { on } \partial \Omega \backslash \overline{D_{1} \cup D_{2}}
\end{array}\right.
$$

Finally, let us discuss the assignment of conductivity values to different tissues inside the realistic human body model. Conductivity image reconstruction from a living human body has been a long-term goal in the area of electrical impedance tomography (EIT) whose corresponding mathematical problem is the inverse problem of identifying the elliptic coefficient from Dirichlet-to-Neumann map. In EIT, elegant mathematical theory and reconstruction algorithms have been developed $[4,5,9,15,16,21,26]$. However, in clinical environments, EIT has not reached yet to the stage providing reliable static conductivity values due to its ill-posed nature.

Recently, magnetic resonance electrical impedance tomography (MREIT) has been proposed to deal with the ill-posed nature of EIT [7, 8, 10,12,17-19,22,24,25]. MREIT uses an MRI system to get a supplementary data, that is one component $B_{z}$ of the magnetic flux density $\mathbf{B}=\left(B_{x}, B_{y}, B_{z}\right)$, where $\mathbf{B}$ is governed by Ampère law $\nabla \times \mathbf{B}=-\sigma \nabla u$ and $u$ is the solution of the elliptic PDE: $\nabla \cdot(\sigma \nabla u)=0$ with an injected Neumann data (or current). Numerous numerical simulations and experiments have shown that MREIT can produce highresolution conductivity images, provided that we inject enough amount of current (or $L^{1}$-norm of Neumann data).

In our future experimental studies to validate numerical simulation results, we plan to first perform conductivity imaging of animal subjects using MREIT techniques. Once we obtain high-resolution conductivity images, then we can generate current density images by solving the direct problem with various position of darts using the method described in this paper. This kind of experimental validations of our numerical method using animal experiments will be invaluable for the interpretation of any numerical results using a realistic human body model since experimental studies using living human subjects are not possible.

Acknowledgements. This work was supported by the SRC/ERC program of MOST/KOSEF (R11-2002-103 and R012005-000-10339-0). Much of this work was done during the visit of IMA, University of Minnesota. We would like to thank IMA. We would like to thank Professor John G. Webster at the Department of Biomedical Engineering, University of Wisconsin-Madison for introducing us this interesting problem and providing valuable technical information on EMD devices. We would like to also thank Professor Min Suk Chung at the Department of Anatomy, Aju University School of Medicine, Korea for providing us the Visible Korean Human data set.

\section{REFERENCES}

[1] G. Alessandrini and R. Magnanini, The index of isolated critical points and solutions of elliptic equations in the plane. Ann. Scoula. Norm. Sup. Pisa Cl. Sci. 19 (1992) 567-589.

[2] G. Alessandrini, E. Rosset and J.K. Seo, Optimal size estimates for the inverse conductivity poblem with one measurement. Proc. Amer. Math. Soc. 128 (2000) 53-64.

[3] Amnesty International, Internet site address: http://web.amnesty.org/library/index/engamr510302006

[4] M. Cheney, D. Isaacson and J.C. Newell, Electrical impedance tomography. SIAM Rev. 41 (1999) 85-101.

[5] V. Isakov, On uniqueness of recovery of a discontinuous conductivity coefficient. Comm. Pure Appl. Math. 41 (1988) 856-877.

[6] P.J. Kim and W.H. Franklin, Ventricular Fibrillation after Stun-Gun Discharge. N. Engl. J. Med. 353 (2005) 958-959. 
[7] S.W. Kim, O. Kwon, J.K. Seo and J.R. Yoon, On a nonlinear partial differential equation arising in magnetic resonance electrical impedance tomography. SIAM J. Math. Anal. 34 (2002) 511-526.

[8] Y.J. Kim, O. Kwon, J.K. Seo and E.J. Woo, Uniqueness and convergence of conductivity image reconstruction in magnetic resonance electrical impedance tomography. Inverse Probl. 19 (2003) 1213-1225.

[9] R. Kohn and M. Vogelius, Determining conductivity by boundary measurements. Comm. Pure Appl. Math. 37 (1984) 113-123.

[10] O. Kwon, E. Woo, J.R. Yoon and J.K. Seo, Magnetic resonance electrical impedance tomography (MREIT): simulation study of J-substitution algorithm. IEEE Trans. Biomed. Eng. 49 (2002) 160-167.

[11] D. Laur, Excited delirium and its correlation to sudden and unexpected death proximal to restraint (Canada: Victoria Police Department) http://www.taser.com/facts/medical_info.htm (2004).

[12] B.I. Lee, S.H. Oh, E.J. Woo, S.Y. Lee, M.H. Cho, O. Kwon, J.K. Seo and W.S. Baek, Static resistivity image of a cubic saline phantom in magnetic resonance electrical impedance tomography (MREIT). Physiol. Meas. 24 (2003) 579-589.

[13] D.K. Mcbride and N.B. Tedder, Efficacy and Safety of Electrical Stun Devices, A Potomac Institute for Policy Studies Report: No. 05 . 04, http://www.potomacinstitute.com/research/Stun\%20Devices\%20Report_FINAL.pdf (2005).

[14] W.C. Mcdaniel, R.A. Stratbucker, M. Nerheim and J.E. Brewer, Cardiac Safety of Neuromuscular Incapacitating Defensive Devices. PACE Supplement 1 (2005) 284-287.

[15] P. Metherall, D.C. Barber, R.H. Smallwood and B.H. Brown, Three Dimensional Electrical Impedance Tomography. Nature 380 (1996) 509-512.

[16] A. Nachman, Reconstructions from boundary measurements. Ann. Math. 128 (1988) 531-577.

[17] S.H. Oh, B.I. Lee, E.J. Woo, S.Y. Lee, M.H. Cho, O. Kwon and J.K. Seo, Conductivity and current density image reconstruction using harmonic $B_{z}$ algorithm in magnetic resonance electrical impedance tomography. Phys. Med. Biol. 48 (2003) 3101-3016.

[18] S.H. Oh, B.I. Lee, S.Y. Lee, E.J. Woo, M.H. Cho, O. Kwon and J.K. Seo, Magnetic resonance electrical impedance tomography: phantom experiments using a 3.0 Tesla MRI system. Magn. Reson. Med. 51 (2004) 1292-1296.

[19] C. Park, O. Kwon, E.J. Woo and J.K. Seo, Electrical conductivity imaging using gradient $B_{z}$ decomposition algorithm in magnetic resonance electrical impedance tomography (MREIT). IEEE Trans. Med. Imag. 23 (2004) 388-394.

[20] J.S. Park, M.S. Chung, S.B. Hwang, Y.S. Lee, D.H. Har and H.S. Park, Visible Korean Human: Improved Serially Sectioned Images of the Entire Body. IEEE Trans. Med. Imag. 24 (2005) 352-360.

[21] F. Santosa and M. Vogelius, A backprojection algorithm for electrical impedance imaging. SIAM J. Appl. Math. 50 (1990) 216-243.

[22] G.C. Scott, M.L.G. Joy, R.L. Armstrong and R.M. Henkelman, Measurement of nonuniform current density by magnetic resonance. IEEE Trans. Med. Imag. 10 (1991) 362-374.

[23] J.K. Seo, A uniqueness results on inverse conductivity problem with two measurements. J. Fourier Anal. App. 2 (1996) $515-524$.

[24] J.K. Seo, J.R. Yoon, E.J. Woo and O. Kwon, Reconstruction of conductivity and current density images using only one component of magnetic field measurements. IEEE Trans. Biomed. Eng. 50 (2003) 1121-1124.

[25] J.K. Seo, O. Kwon, B.I. Lee and E.J. Woo, Reconstruction of current density distributions in axially symmetric cylindrical sections using one component of magnetic flux density: computer simulation study. Physiol. Meas. 24 (2003) 565-577.

[26] J. Sylvester and G. Uhlmann, A global uniqueness theorem for an inverse boundary value problem. Ann. Math. 125 (1987) 153-169.

[27] Taser M26 and X26 manuals, http://www.taser.com/index.htm

[28] G. Verchota, Layer potentials and boundary value problems for Laplace's equation in Lipschitz domains. J. Func. Anal. 59 (1984) $572-611$.

[29] J.G. Webster, Electromuscular Incapacitating Devices. Proc. IFMBE 20059 (2005) 150-151. 